\title{
Application of reverse osmosis and nanofiltration techniques at municipal drinking water facilities
}

\author{
Alexei Pervov* and Xuan Quyet Nguyen \\ Moscow State University of Civil Engineering, Yaroslavskoe shosse, 26, Moscow, 129337, Russia
}

\begin{abstract}
Main disadvantages of state of the art membrane techniques are discussed with respect to the problem of concentrate disposal. Possible ways to improve conventional membrane techniques and to radically decrease the number of effluents discharged are proposed. Application of newly developed "open channel" membrane modules that do not contain "dead" areas enables us to develop and implement into practice new techniques to reduce and even utilize concentrates of membrane units. Experiments demonstrated a possibility to reduce concentrate flow due to deposition of excessive calcium as calcium carbonate on seed crystals in the reactor. Flow diagrams for different cases of natural water composition are described and discussed that demonstrate newly developed techniques to produce drinking quality water using reverse osmosis and nanofiltration membrane facilities with high recoveries.
\end{abstract}

\section{Introduction}

The ability of membrane to simultaneously reject a number of dissolved different impurities (such as: organics, colour, calcium, magnesium, pesticides, ammonia etc.) offers a new solution to production of quality water production. Meanwhile, long industrial application experience demonstrated that reverse osmosis membrane facilities require very proper pretreatment and maintenance that includes chemical dosing, cleanings, as well as reject (concentrate) stream handling. These measures are essential to prevent fouling and scaling on membrane surface during its operation [1-4]. Thus, when quality water is produced using membranes, their maintenance costs often exceeds costs required to treat water by conventional techniques, that involve flocculation, filtration, sorption and ion exchange process. This turns advantages of membrane techniques over conventional tools into "disadvantages". When municipal water treatment facilities are developed, water recovery (the ratio between raw water flow and purified water flow) becomes a main decisive factor. High values of concentrate flow (that constitute up to 25 -35 per cent of raw water flow) are the main disadvantage of membrane units are required to ensure their safe operation and escape scaling of low soluble deposits (mainly calcium carbonate) on membrane surface $[5,6]$. Reduction of concentrate flow values and "utilization" of concentrate still remains

\footnotetext{
* Corresponding author: ale-pervov@yandex.ru
} 
main challenge that requires scientific solution [7, 8]. The main "key" to this solution is efficient control of scaling when treating of natural water with recovery values that exceed supersaturation limits of calcium carbonate [9, 10]. Previously it was reported that elimination of "dead areas" that occur in membrane channels of existing conventional membrane modules provides reduction of fouling and scaling as well as recovery increase [11-13]. A new module configuration proposed an "open" membrane channel that does not contain turbulization mesh that created "dead areas" on membrane surface, increased hydraulic resistance and "trapped" particles suspended in the flow [11]. Calcium carbonate and calcium sulphate supersaturated solutions are very stable and do not demonstrate visual nucleation even at high supersaturation values $[14,15]$. Therefore, the use of new modules with "open channels" that are deprived of the "dead areas" responsible for scale formation enables us to operate membrane facilities at high recovery values without scaling. These modules with "open channel" possess very low fouling propensities as flow resistance to the flow does not exist. Fouling layers are efficiently and easily destroyed and withdrawn from membrane channel through the use of timely "flushings" [16]. Similar operation mode distinguishes tubular membrane modules, but tubular membranes demonstrate high capital and operational costs [17].

Present article is aimed at development of new membrane facilities with decreased concentrate flow due to efficient softening without reagent use. Principles of excessive calcium removal from reverse osmosis concentrate are demonstrated on Figure 1. Concentrate flow after reverse osmosis unit enters reactor with "seed" crystals of already precipitated calcium carbonate. The membrane unit is operated in circulation mode and concentrate after passing through reactor is returned back to the access to the membrane unit. Thus, calcium and bicarbonate ions are constantly concentrated in membrane unit and concentrate that enters reactor is always supersaturated. Supersaturation is followed by crystal nucleation and growth. Reduction of calcium and carbonate ion concentration due to seeded crystal growth enables us to increase membrane unit recovery.

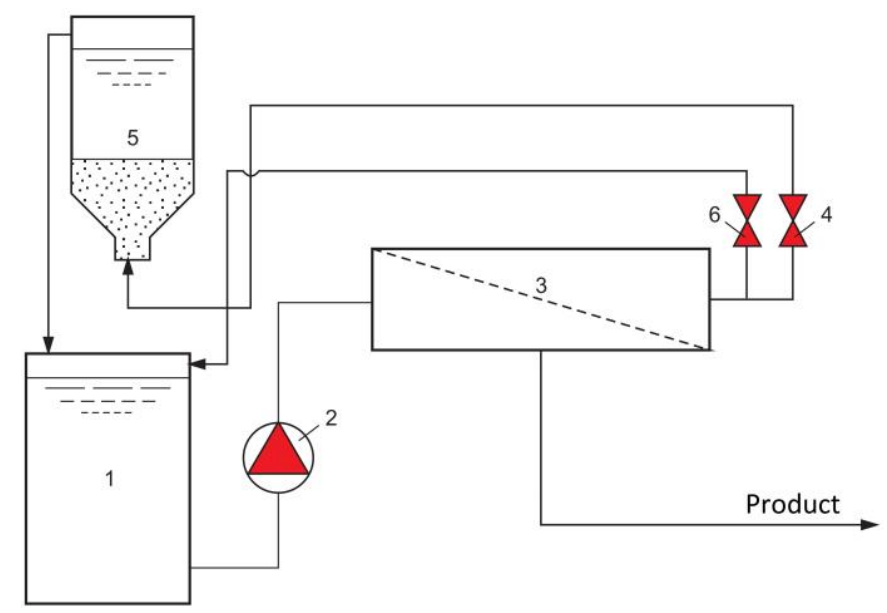

Fig. 1. Supersaturation is followed by crystal nucleation and growth.

1 - feed water tank; 2 - centrifugal pump; 3 - membrane module;

4 - pressure regulation valve; 5 - reactor; 6 - bypass valve

\section{Experimental procedure}

Membrane test unit flow diagram is presented on Figure 1. Feed water is added to feed water tank 1 . The volume of the feed water was 60 liters. Feed water from tank 1 is pumped 
by centrifugal pump 2 to membrane module 3 . In membrane module feed water was separated into product water and concentrate. Product stream was constantly withdrawn from the test unit and concentrate stream was returned back to the reservoir 1. Concentrate flow value was regulated by the valve 4 . To withdraw calcium carbonate from concentrate stream a "seed" reactor 5 was used. Calcium carbonate seed crystals were suspended in the up-flow stream that passed through reactor. Calcium carbonate (calcite) crystals were were formed by addition of stoichmetric amount of sodium hydroxide to 60 liters of feed water and further sedimentation. Calcium hardness of the feed water was 4 milliequivalents per liter. Concentrate enters the lower part of reactor and overflows back into the reservoir 1 . Concentrate flow value was regulated by the valve 6 on bypass line. Centrifugal pump Speroni RSM-5 (Italy) was used that provided a flow of 10 liter per minute under 8Bar pressure. Membrane modules were manufactured by the authors using CSM membranes BLN type. The modules were manufactured according to 1812 standard: 1,8 inch $(36 \mathrm{~mm})$ in diameter and 12 inch $(300 \mathrm{~mm})$ length. Membrane area was 0,5 square meters. Moscow tap water was used in experiments. To initiate crystal formation, sodium hydroxide solution $(40 \%)$ was used.

Table 1

\begin{tabular}{|l|c|c|c|c|c|}
\hline \multicolumn{1}{|c|}{ Parameter } & $\begin{array}{c}\text { Feed } \\
\text { water }\end{array}$ & $\begin{array}{c}\text { Concentrate, } \\
\text { Concentrate } \\
\text { (recovery 90\%) }\end{array}$ & $\begin{array}{c}\text { passed through a } \\
\text { layer of seed } \\
\text { crystals } \\
\text { (recovery 90\%) }\end{array}$ & Product & $\begin{array}{c}\text { The mixture } \\
\text { of filtrate } \\
\text { and } \\
\text { concentrate }\end{array}$ \\
\hline Calcium, mEq/L & 4,6 & 38,6 & 10,6 & 0,32 & 1,3 \\
\hline $\begin{array}{l}\text { Total hardness, } \\
\mathrm{mEq} / \mathrm{L}\end{array}$ & 6,7 & 57,8 & 33,4 & 0,71 & 4,1 \\
\hline $\mathrm{Chloride,} \mathrm{mEq} / \mathrm{L}$ & 37,9 & 331 & 323 & 16,4 & 49,1 \\
\hline $\mathrm{HCO}_{3}, \mathrm{mEq} / \mathrm{L}$ & 6,1 & 47,6 & 17,1 & 1,4 & 1,9 \\
\hline $\mathrm{pH}$ & 6,8 & 8 & 7,9 & 6,1 & 6,7 \\
\hline $\begin{array}{l}\mathrm{Carbonate} \text { index, } \\
(\mathrm{mEq} / \mathrm{L})^{2}\end{array}$ & 28,06 & - & - & 0,44 & 2,42 \\
\hline
\end{tabular}

Table 2

\begin{tabular}{|l|c|c|c|}
\hline \multicolumn{1}{|c|}{ Parameter } & $\begin{array}{c}\text { Feed (underground) } \\
\text { water }\end{array}$ & $\begin{array}{c}\text { Concentrate } \\
\text { (recovery 60\%) }\end{array}$ & $\begin{array}{c}\text { Concentrate after } \\
\text { contact with seed } \\
\text { crystals }\end{array}$ \\
\hline $\mathrm{Ca}^{2+}, \mathrm{mEq} / \mathrm{L}$ & 3,75 & 8,2 & 4 \\
\hline Bicarbonate, $\mathrm{mEq} / \mathrm{L}$ & 4,1 & 9,4 & 5,2 \\
\hline Chloride, $\mathrm{mEq} / \mathrm{L}$ & 9,23 & 21,3 & 21,3 \\
\hline $\mathrm{pH}$ & 6,8 & 7,87 & 7 \\
\hline
\end{tabular}

During the test unit operation the volume of the feed water in tank 1 decreased as product water was withdrawn from the system. As a result, concentration values of calcium, magnesium, bicarbonate and carbonate ions as well as TDS constantly increased (Table 1). Therefore, concentrate became supersaturated providing high potential for calcium carbonate deposition. "Seed" mass in reactor constantly increased due to calcium carbonate crystal growth. The first experimental series was aimed at determination of "seed"crystal growth rates in different conditions. The feed water volume was reduced by 6 times (from 60 liters to 10 liters). Stoichmetric amounst of caustic (250 milliequivalents) were added to 
concentrate, deposited calcium carbonate (calcite) crystals were later sedimented and collected. New portion of concentrate (20 liter volume) was produced from 60 liters of feed water, and the deposited previously "seed" slurry was added to concentrate. Reduction of calcium, chloride and bicarbonate ion concentration values with time are shown in Table 2.

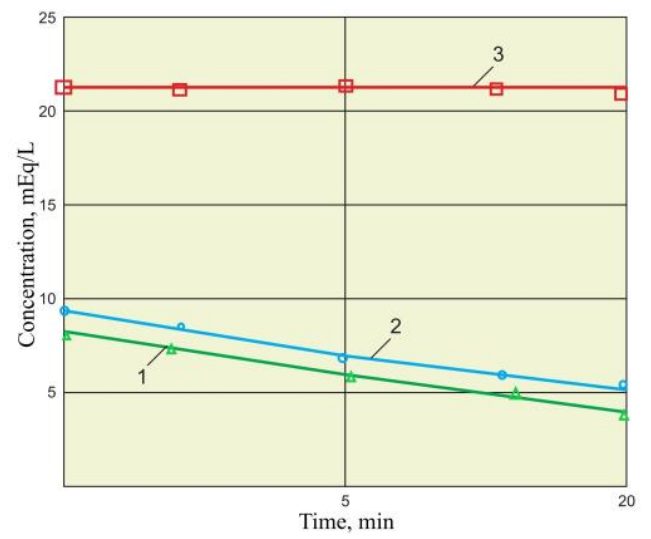

Fig.2. Reduction of calcium and bicarbonate ion concentration with time after addition of seed mass to concentrate. 1 - calcium concentration; 2 - bicarbonate concentration; 3 - chloride concentration.

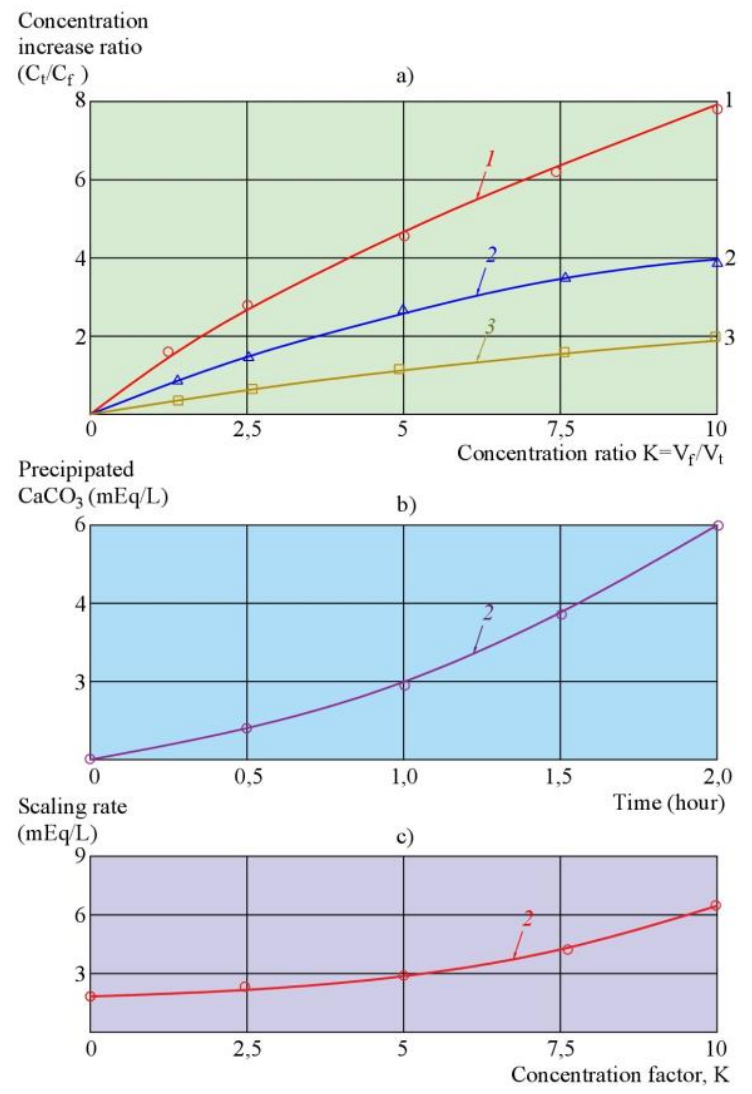

Fig. 3. Dependencies of calcium, chloride and bicarbonate ion concentration values versus $\mathrm{K}$ (concentration factor) in concentrate after contact with seed mass at the exit of reactor 1 - chlorides; 2 - bicarbonate; 3 - calcium 
Figure 2 demonstrates behaviour of calcium, bicarbonate and chloride ion concentration values with time after contact of concentrate with seed mass.

It is obvious that calcium carbonate crystallization rate rapidly decreases as well as supersaturation value as consumption of carbonate ions be growing crystals causes the increase o $\mathrm{pH}$ value $[14,15]$. To ensure constant crystal growth in the "seed" reactor, high supersaturation values should be provided. This was implemented by operation of the test unit in circulation mode whereby concentrate was returned back to the tank 1 and product water was constantly withdrawn (Figure 1). Calcium and carbonate ion concentration values therefore constantly increased in concentrate of the test unit throughout the test run.

Process of calcium carbonate crystallization is illustrated on Figure 3. Figure 3 (a) shows dependencies of calcium ion concentration values on the concentration factor $\mathrm{K}$ ( $\mathrm{a}$ ratio of the feed water initial volume to the volume at the certain moment during experiment, $(\mathrm{K}=\mathrm{Vf} / \mathrm{Vt}))$. The amount of seed crystals in reactor facilitates crystallization. Curves 1 and 2 (Figure 3) demonstrate increase of calcium concentration in the circulated feed water solution without contact with seed mass. This shows possible crystallization process in membrane module due to "dead areas". Thus, crystallization rate of the seed mass is dependent on supersaturation and amount of seeds. Figure 3(b) shows dependencies of mass of calcium carbonate deposited on seed crystals versus time elapsed from the beginning of experiment. Crystal growth rates at different experiment moments were determined as values of tangents of the experimental curves. As reached volumes of concentrate throughout experiment corresponded to time values elapsed from the beginning of experiments, we developed finally dependencies of calculated calcium carbonate scaling rate values on concentration factor $\mathrm{K}$ (Figure 3, c).

\section{Discussion of the results}

Sparingly soluble salts deposition kinetics is described in a number of publications [11-15]. Nucleation (formation of crystal nuclei) phase always precedes crystal growth. This phase consists of formation of nuclei that further act as "crystallization centres". The amount of nuclei formed in the unit volume at the unit time is considered as homogeneous nucleation rate is dependent on supersaturation value. In our case the amount of nuclei formed depends on supersaturation value achieved after caustic addition.

The obtained experimental results show, that addition of even small amount of seed crystals to concentrate enables us to precipitate large amount of calcium carbonate that exceeds seed amount by 15-20 times. Table 1 shows calcium concentration values throughout experiments. After initial volume of feed water was decreased by 10 times calcium concentration value increased from 4,6 to 11,6 milliequivalents per liter. Then we blended softened concentrate with product water collected in separate product tank. Results of blending are shown in Table 1. After blending we obtained a solution that contained 1,3 milliequivalents per liter calcium and 1,9 milliequivalents of alkalinity (bicarbonate). TDS value of the blend was $200 \mathrm{ppm}$ lower than of the initial feed water. Thus, the developed technique of concentrate softening using seed crystals enables us to produce softened water from natural water with high hardness. This softening process does not require stoichmetric amounts of reagents and does not produce saline effluents. Figure 4 suggests principles of quality drinking water production from natural water showing flow diagrams and mass balance calculations for different cases of natural water compositions. The distinguishing feature of new proposed technique is the use of membrane process as a "driving force" for calcium carbonate supersaturation and further deposition. In "dead "areas of membrane channels calcium and carbonate ion concentrations can increase by 20-30 times [11] that is 

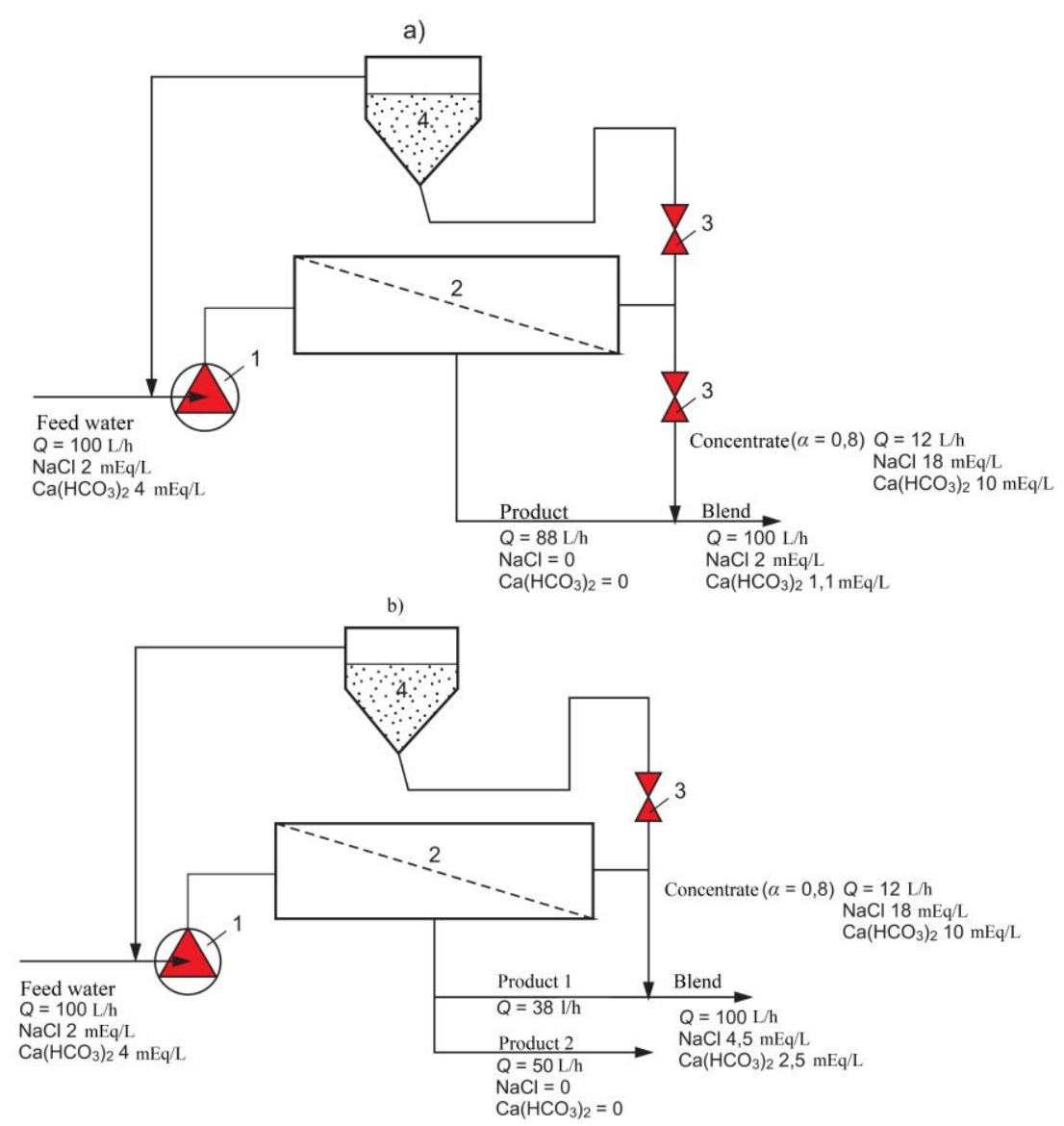

c)

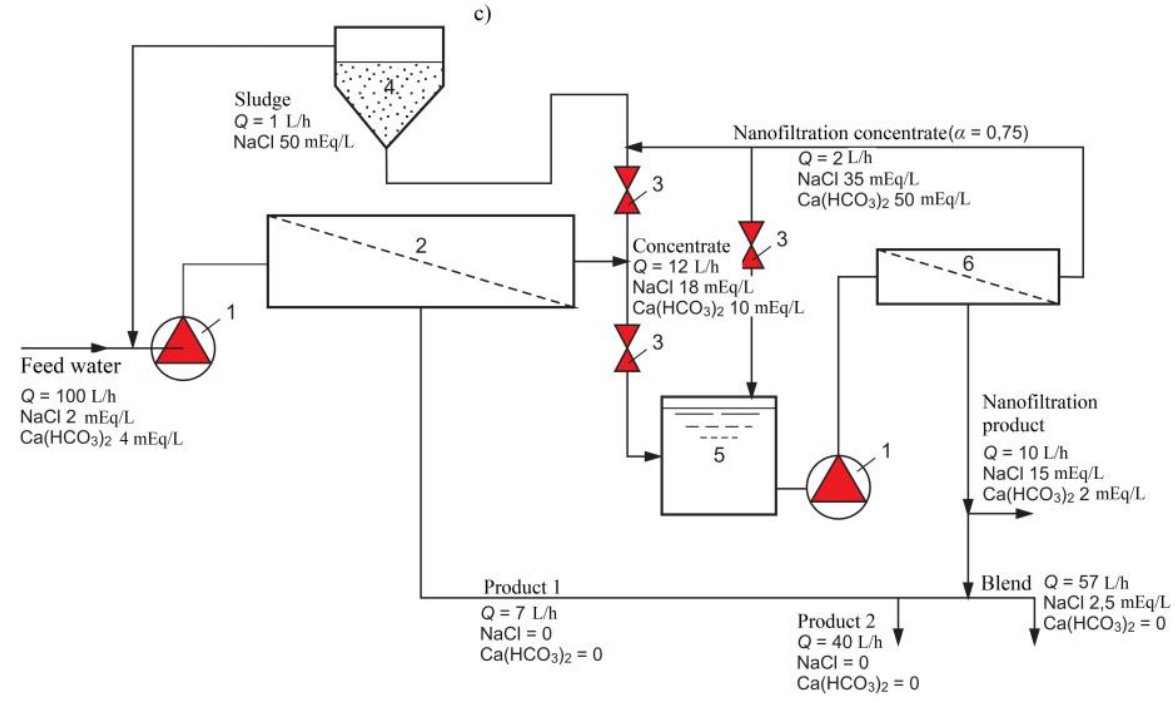

Fig. 4. Flow diagrams and mass balances of water treatment processes that use seed precipitation reactor; $a$ - blending of concentrate with product water; $b$ - blending of a part of concentrate with product water; $\mathrm{c}$ - treatment of concentrate with nanofiltration unit;

1 - centrifugal pump; 2 - reverse osmosis membrane module; 3 - pressure regulation valve; 4 - seed precipitation reactor; 5 - concentrate accumulation tank; 6 - nanofiltration module 
considered as a main reason for beginning of homogeneous nucleation and further crystal formation. The described calcium deposition technique does not use sodium hydroxide to create supersaturation and to deposit all calcium carbonate contained in feed water, but merely to produce seed crystals. Further growth of these crystals occurred due to supersaturation reached in circulated concentrate of membrane unit due to product water withdrawal.

\section{Industrial application of results}

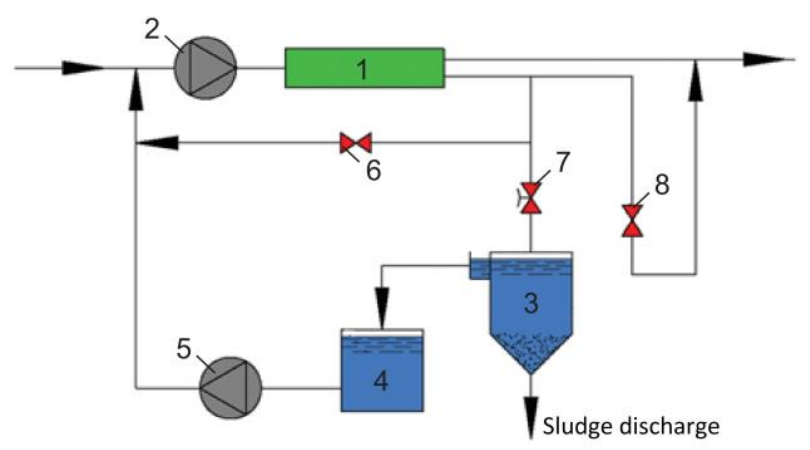

Fig. 5. A flow diagram of production of quality drinking water with nanofiltration membranes with concentrate utilization. 1 - membrane module; 2 - centrifugal pump; 3 - sedimentation tank to collect "flush" water; 4 - product water reservoir; 5 - clarified water pump ; 6 - bypass valve; 7 solenoid valve; 8 - pressure regulation valve

The developed approach to reduce concentrate flow of membrane facilities could be used at water treatment facilities that produce drinking water. A flow diagram of surface water treatment plant that uses nanofiltration facilities is presented on Figure 5. Membrane unit is operated in circulation mode that ensures high recovery value of 85-95 percent. Suspended particles that are contained in the feed water can foul membrane. The dissolved organics (fulvic and humic acids) adhere to membrane surface and foulant particles $[11,16]$.

Table 3.

\begin{tabular}{|c|c|c|c|c|}
\hline Parameter & $\begin{array}{c}\text { "Desna" } \\
\text { river } \\
\text { water }\end{array}$ & Product & Concentrate & Blend \\
\hline Calcium, mEq/L & 3,8 & 0,5 & 9,5 & 1,85 \\
\hline Magnesium, mEq/L & 2,3 & 0,5 & 12,5 & 2,3 \\
\hline Chloride, ppm & 72,4 & 17,5 & 364 & \\
\hline Sulphate, ppm & 39,7 & 10,0 & 208 & 39,6 \\
\hline Alkalinity (hydrocarbonate), \\
mEq/L & 5,9 & 2,2 & 20,5 & 4,94 \\
\hline pH & 6,75 & 6,04 & 7,6 & 6,5 \\
\hline Oxidity, ppm & 6,48 & 1,52 & 11,8 & 3,0 \\
\hline Colour, NTU & 42 & 7,5 & 91 & 20,0 \\
\hline Turbidity, ppm & 12,1 & 0,5 & 3,5 & 1,5 \\
\hline
\end{tabular}


Application of hydraulic "flushing" procedures destroys fouling layer and removes particles from membrane surface. Foulant removed from membrane surface is forwarded as a "flush" water to sedimentation tank. Sedimented sludge further dewatered and reject water after dewarering is added to the feed water. Thus the dewatered sludge contains all impurities (suspended and colloidal matter and organics) removed by membrane. Concentrate of membrane unit can be mixed with product water and main characteristics of the blended water (suspended matter, colour and oxidity) meet drinking water WHO (World Health Organization) standards. Table 3 shows results of the surface water (Desna River), product water and concentrate chemical analysis as well as results of blending. The recovery of the pilot plant that treated surface water was $85 \%$.

The sedimented sludge therefore contains main impurities (suspended and colloidal matter and organics) removed by membrane. Concentrate of membrane unit can be mixed with product water and main characteristics of the blended water (suspended matter, colour and oxidity) meet drinking water WHO (World Health Organization) standards. Table 3 shows results of the surface water (Desna River), product water and concentrate chemical analysis as well as results of blending. The recovery of the pilot plant that treated surface water was $85 \%$.

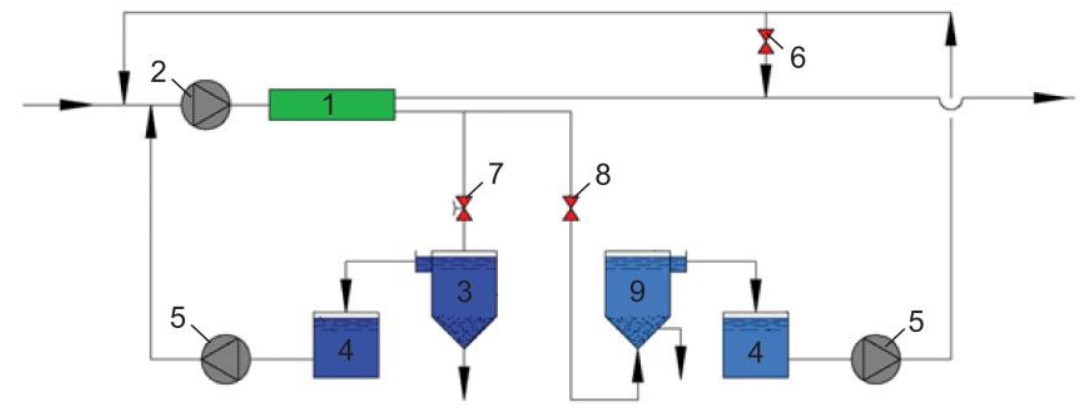

Fig. 6. A flow diagram of ground water treatment plant that use reverse osmosis facilities and "seed" reactor to reduce concentrate. 1 - membrane module; 2 - centrifugal pump; 3 - sedimentation tank to collect "flush" water; 4 -clarified water tank; 5 - clarified water pumps; 6 - bypass valve; 7 - solenoid valve; 8 - pressure regulation valve; 9 - seed precipitation reactor

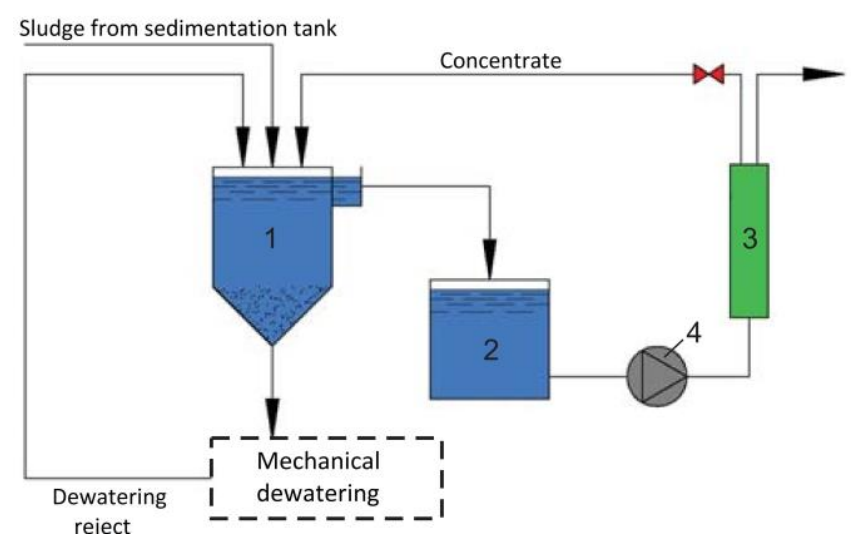

Fig.7. A flow diagram of treatment of reject water after sludge dewatering using reverse osmosis techniques. 1- sludge "thickening" sedimentation tank; 2 - clarified water tank; 3 - membrane module; 4 - centrifugal pump 
The described techniques provide efficient solution to remove organics, colloidal matter and iron from natural water for drinking water production . For the cases where additional softening, as well as removal of fluoride, ammonia, strontium and other impurities are required, similar techniques can be applied. But in these cases only a part of concentrate should be blended with product water. The other part of concentrate volume should be be reduced by 30-100 times and mixed with the wet sludge that is further forwarded to dewatering. Sludge moisture ( amount of water in dewatered sludge usually constitutes $0,7-1,0$ percent of the initial feed water flow. All impurities rejected by membranes can be withdrawn together with the dewatered sludge as the sludge moisture. The reject water after sludge dewatering can be mixed with the feed water that enters membrane unit. Application of the described above calcium carbonate deposition from concentrate enables us to reduce feed water TDS by 30-40 per cent and to reduce volume of concentrate by 100-200 times. For example, for feed water TDS value of 300-500 ppm, concentrate TDS values reach 30000-50000 ppm.

Figure 6 shows a flow diagram of treatment of natural water using membranes for the cases when concentrate flow is reduced by means of "seed" precipitation and further removed together with the sludge.

The described above tools to reduce concentrate flow can be used at the existing water treatment conventional facilities to utilize reject water after sludge dewatering. This reject contains large amount of aluminum, high molecular organics (fulvic and humic acids), flocculants. Usually this reject is forwarded to the sewer as it is not easy to purify it efficiently to meet drinking water standards or discharge regulations. Using membrane techniques with "open channels" the reject stream can be purified. Concentrate of membrane unit should constitute 5-10 per cent of reject stream. Concentrate is mixed with the wet sludge. The flow diagram of the process is shown on Figure 7. Calculations of concentrate flow rate and prediction of it's chemical composition can be provided by means of the mass balance between the amount of impurities in the wet sludge and in dewatered sludge. Product water of membrane is mixed with drinking water or used for technical needs.

\section{Conclusion}

The existence of concentrate streams increase operational costs of membrane water purification facilities, complicates membrane maintenance, creates a problem of concentrate handling and disposal and often becomes a reason why efficient membrane techniques are refused to be applied.

High concentrate flow values are explained by precautions not to exceed supersaturation limits of calcium sulphate and calcium carbonate in concentrate streams.

The described techniques to increase recovery are based on withdrawal of excessive calcium carbonate from concentrate. This approach does not use chemicals to deposit calcium carbonate. Calcium carbonate is deposited on the "seed" crystals due to supersaturation reached in concentrate of membrane modules.

Concentrate flow can be reduced by $95-100$ times and constitute no more than $1 \%$ of the feed water flow.

Concentrate can be mixed with wet sludge and all impurities that are contained in concentrate are gischarged together with dewatered sludge as a sludge moisture.

This work was supported by research grant № 19-08-00982 of Research Foundation of Basic Research. 


\section{References}

1. S. Jamaly, N.N., Darwish, I. Ahmed, S.W. Hasan, Desalination 354, 30-38 (2014)

2. P.S. Goh, W.J. Lau, M.H.D. Othman, A.F. Ismail, Desalination, 425, 104-111 (2018)

3. C. Fritzmann, J. Lowenberg, T. Wintgens, T. Melin, Desalination, 216, 1-76 (2007)

4. L.Henthorne, B. Boysen, Desalination, 356, 129-139 (2015)

5. L. Malaeb, G.M. Ayoub, Desalination 267, 1-8 (2011)

6. G.D. Kang, Y.M. Cao, Water Research, 46, 584-600 (2012)

7. Y.M. Al-Roomi, K.F. Hussain, Desalination 393, 186-195 (2016)

8. A.Rahardianto, W.-Y. Shih, R.-W.Lee, Y.Cohen, Journal of Membrane Science, 279, 655-668 (2006)

9. M. Turek, K. Mitko, P. Dydo, E. Laskowska, A. Jacobic-Kolon, Desalination, 401, 180-189 (2017)

10. Srinivas Veespareni, Rich Bond. Tianjin IDA World Congress 2013 on Desalination and Water Reuse, TIAN 13-357 (October 20 -25, China 2013)

11. A.G. Pervov, A.P. Andrianov, Desalination and wastewater treatment 35, 2-9 (2011)

12. A. Pervov, Desalination, 368, 140-151 (2015)

13. Alexei Pervov, Alexei Andrianov, Desalination and Water Treatment, 1-9 (2018)

14. Motohiro Okazaki and Shoji Kimura, Desalination, No. 21 (1977)

15. Nancollas G.H., Advances in colloid and interface science. No. 10 (1979)

16. A.G. Pervov, Desalination, 126, 227-247 (1999)

17. Mark Wilf. The guidebook to membrane for wastewater reclamation. Balaban Desalination Publications, 788 (2010) 